\title{
On Optimized Color Image Coding Using Correlation of Primary Colors
}

\author{
Eyal Braunstain ${ }^{1}$ and Moshe Porat ${ }^{2}$ \\ ${ }^{1}$ Department of Biomedical Engineering \\ Technion - Israel Institute of Technology, Haifa 32000, Israel \\ seyalbraatx.technion.ac.il \\ ${ }^{2}$ Department of Electrical Engineering \\ Technion - Israel Institute of Technology, Haifa 32000, Israel \\ mpeee.technion.ac.il
}

\begin{abstract}
The RGB color primaries in natural images are characterized by a high degree of inter-correlation. Many compression algorithms use this information redundancy to reduce the amount of bits required for coding, by transforming the color information to a decorrelated color space - such as YUV. The human visual system is more sensitive to luminance than chrominance components, so more bits are allocated to luminance. We examine a different approach, by expressing two of the color components (termed subordinate colors) as a functional of the other color component (termed base color). Unlike some compression algorithms (e.g. JPEG) that perform the analysis on $\mathrm{NxN}$ blocks in the image, we utilize segmentation by Region Growing in both gray level and color (RGB) images. We suggest a method for selection of optimal base color for each region separately. The proposed approach could be useful for color compression and progressive transmission applications.
\end{abstract}

Keywords: Color compression, Correlation, Polynomial approximation.

\section{Introduction}

Natural images are characterized by a high degree of correlation between the color primaries (RGB). This inter-dependence yields the possibility of reducing the amount of data required for representation, e.g. by compression algorithms that transform the color components to a decorrelated color space, e.g. YUV (Y - luminance component, $\mathrm{U}, \mathrm{V}$ - chrominance), and utilizing the fact that the human visual system is more sensitive to details in luminance than chrominance, and encode the luminance information using more bits. Another approach to utilizing the correlation between the color primaries (RGB) is by expressing two of the color components, termed 'subordinate colors' as a function of a third color component, termed 'base color' [1], [2].

In many compression algorithms, e.g., JPEG, the image is divided into $\mathrm{NxN}$ - pixels blocks, without reference to the spatial correlation between adjacent pixels. In the proposed approach, we refer to image segments, acquired by Region Growing 
segmentation [3], [4]. More advanced segmentation methods can be utilized, e.g. Quick Shift [5]. In methods that divide the image into arbitrary NxN blocks, distorting blockiness artifacts are created in the compressed image, which can be diminished by segmentation into natural regions. Region Growing can be performed on a gray level image, using a simple absolute distance to grow regions from seeds. However, performing the segmentation with full color information, rather than relying on brightness alone, can yield superior segmentation results. Therefore, we examine the compression results achieved when the Region Growing is performed on an RGB color image, using Euclidean metric to grow regions from seeds. This approach generally provides better segmentation, which results in superior reconstruction (decompression) results, both visually and by PSNR (Peak Signal to Noise Ratio) measure.

The image segments (texture) and region boundaries (contours) are encoded separately. The texture information of each segment is encoded using a base color, and by expressing the subordinate colors as functions ( 0 or $1^{\text {st }}$ order) of this base color. The base color itself is encoded as a $0 / 1^{\text {st }} / 2^{\text {nd }}$ order polynomial approximation of the actual base color pixels in the segment. In [1], the authors propose to compress an image by using $\mathrm{NxN}$ pixels blocks, and in each block express two subordinate colors as a function of a base color, which can be chosen variably for each block by an optimization method, according to the highest correlation of a base color to the other two colors, or by choosing the base color that leads to the lowest subordinate colors' reconstruction error for the block. We propose to choose the base color that yields the minimal reconstruction error (MSE) of both the base and subordinate approximated colors for a region. Our proposed approach, based on image segmentation by color Region Growing and optimal base color selection for the utilization of intercorrelation of colors, can lead to significant improvement of compression results, compared to the widespread JPEG compression algorithm, especially for high compression ratios, as shown by our experimental results.

\section{$2 \quad$ Methodology}

The compression algorithm is comprised of encoder and decoder blocks. The stages of the encoder block are as follows:

1. Construct the luminance image (Y) from the $\mathrm{R}, \mathrm{G}$, and $\mathrm{B}$ color primaries:

$$
\mathrm{Y}=0.3 \mathrm{R}+0.59 \mathrm{G}+0.11 \mathrm{~B} \text {. }
$$

2. In order to get a smoother image, and suppress false contours in the luminance image, we filter the $\mathrm{Y}$ image using the inverse gradient filter [6]. This filter reduces the noise in Y image, while preserving contours (high gradients).

3. Perform Region Growing segmentation on the smoothed image. We examine the compression results for both gray level and color (RGB) Region Growing. In gray level Region Growing, a region is grown from a seed pixel by adding to the region neighboring pixels whose gray level is inside a fixed gray level interval from the region mean gray level. Seed pixels are chosen randomly, from pixels not yet assigned to specific regions. In RGB Region Growing, the above gray level interval is replaced by a Euclidean distance in RGB color space, meaning that a new pixel 
will be added to a current region if its (R, G, B) color triplet is distant less than a fixed threshold from the region's current mean ( $R, G, B)$ values. In both Region Growing options, a pixel that is rejected from a region is marked as contour pixel.

The RGB Euclidean metric for Region Growing is defined as follows:

$d_{R G B}=\sqrt{\left(R_{\text {pixel }}-R_{\text {regMean }}\right)^{2}+\left(G_{\text {pixel }}-G_{\text {regMean }}\right)^{2}+\left(B_{\text {pixel }}-B_{\text {regMean }}\right)^{2}}<d_{R G B_{-} \text {Thresh }}$

4. Contour artifacts, e.g. double contours and non-separating contours pixels are removed from the segmentation image. Small regions (having a number of pixels less than threshold size) that are connected through contour with other regions, are removed, i.e., united with a neighbor region with the closest gray level [7].

5. The contour information is encoded by chain coding [8]. The contour encoding is based on Freeman's chain-code, with 8-connected contours. In natural images' contours, sharp turns are less frequent than gradual turns [7]. Thus, a statistical approach is employed, and the difference in direction between sequential contour pixels is encoded using Huffman coding. The most probable direction of movement is the same as the previous; the next probable direction is a $45^{\circ}$ turn, etc. This enables the reduction of the number of bits required to code the image contours.

6. For each region, a base color is chosen $(\mathrm{R}, \mathrm{G}$, or $\mathrm{B})$, and approximated by a $0 / 1^{\text {st }} /$ $2^{\text {nd }}$ order two-dimensional polynomial function. For example, a $1^{\text {st }}$ order approximation of some region's color by the spatial axes $x$ and $y$, will be of the form $\mathrm{Y}_{\text {approx }}=a_{1}+a_{2} x+a_{3} y$, where $a_{1}, a_{2}, a_{3}$ are the approximation coefficients, and $\mathrm{Y}_{\text {approx }}$ is the approximation of the region color surface $\mathrm{Y}$. The solution for $a_{1}, a_{2}, a_{3}$ is given by the minimization of the square error between $\mathrm{Y}$ and $\mathrm{Y}_{\text {approx }}$, which leads to the solution of the following system of equations [7]:

$$
\left(\begin{array}{ccc}
n & \sum x_{i} & \sum y_{i} \\
\sum x_{i} & \sum x_{i}^{2} & \sum x_{i} y_{i} \\
\sum y_{i} & \sum x_{i} y_{i} & \sum y_{i}^{2}
\end{array}\right) \cdot\left(\begin{array}{l}
a_{1} \\
a_{2} \\
a_{3}
\end{array}\right)=\left(\begin{array}{c}
B C_{i} \\
\sum x_{i} B C_{i} \\
\sum y_{i} B C_{i}
\end{array}\right)
$$

where $x_{i}, y_{i}$ are the image coordinates, and $B C_{i}$ are the base color values of pixels.

A similar derivation exists for $2^{\text {nd }}$ order approximation of base color, by minimization of a square error. Zero-order approximation is given by the region average.

The other two colors (subordinate colors) are encoded by polynomial expansion of the base color for each region [1]:

$$
\begin{aligned}
& C_{1}=a_{k} C_{0}{ }^{k}+a_{k-1} C_{0}{ }^{k-1}+\ldots+a_{1} C_{0}+a_{0} \\
& C_{2}=b_{k} C_{0}{ }^{k}+b_{k-1} C_{0}{ }^{k-1}+\ldots+b_{1} C_{0}+b_{0}
\end{aligned}
$$

where $C_{1}, C_{2}$ are the interpolated subordinate colors, $C_{0}$ is the base color, and $\mathrm{a}_{\mathrm{k}}, \mathrm{b}_{\mathrm{k}}$ are the polynomial expansion coefficients for the subordinate colors.

For $1^{\text {st }}$ order polynomial expansion of subordinate color $\mathrm{R}$ as function of base color $\mathrm{G}$, we have [7]: 


$$
R_{\text {app }}=r_{1} G+r_{0}
$$

The coefficients $r_{i}$ that minimize the mean square error between the original $\mathrm{R}$ component in the region, and the approximated $\mathrm{R}$ component, are hence derived:

$$
g_{0}=\frac{1}{n} \sum_{1}^{n} G_{i} \rightarrow r_{0}=\frac{1}{n} \sum_{1}^{n} R_{i} \quad, \quad r_{1}=\frac{\sum_{1}^{n}\left(R_{i}-r_{0}\right) \cdot\left(G_{i}-g_{0}\right)}{\sqrt{\sum_{1}^{n} G_{i}^{2}}}
$$

The above summation is made upon all pixels in region $i$.

The base color can be fixed - e.g. green, thus determining the subordinate colors to be red and blue. The base color for a region can also be chosen as the color component that has the highest correlation with the other two components, from the color triplet. The approach adopted in this algorithm, is to choose a base color for a region that minimizes the reconstruction error of all three colors in the region - i.e., minimum base and subordinate colors' approximation mean square errors (base color - BC, subordinate color - SC):

$$
\begin{aligned}
& B C_{\mathrm{Re}_{-} i}=\underset{B C \in\{R, G, B\}}{\arg \min }\left\{B C C_{-} \operatorname{Re} c E r r_{\mathrm{Re}_{-} i}+S C_{-} \operatorname{Re} c E r r_{\mathrm{Re}_{g_{-}} i}\right\} \\
& B C_{-} \operatorname{Re} c E r r_{\operatorname{Re} g_{-} i}=\underset{p}{\operatorname{mean}}\left(B C_{p_{-} a p p r o x}-B C_{p}\right)^{2} \\
& S C_{-} \operatorname{Re} c E r r_{\operatorname{Re}_{-} i}=S C_{1} \operatorname{Re} c E r r_{\operatorname{Re}_{g_{-} i}}+S C_{2} \operatorname{Re}_{2} E r r_{\operatorname{Re}_{g_{-} i}} \\
& S C_{k_{-}} \operatorname{Re} c E r r_{\operatorname{Reg}_{-} i}=\underset{p}{\operatorname{mean}}\left(S C_{p_{-} k_{-} a p p r o x}-S C_{p_{-} k}\right)^{2} \quad k=1,2
\end{aligned}
$$

The average over $p$ is for pixels in region i. RecErr refers to MSE reconstruction error. Utilizing other perceptual measures instead of MSE, might cause preference and bias towards specific colors to serve as base colors, which might result in a smaller compression ratio.

7. The coefficients of the optimal base color for each region, and coefficients of polynomial expansion for subordinate colors are quantized.

The stages of the decoder block are as follows:

1. The segmentation contours are reconstructed from the compressed data.

2. Region color texture is reconstructed from base and subordinate color coefficients.

3. The contours pixels are each approximated by the pixels' color components of the surrounding dominant (maximal size) reconstructed segment. The inclusion of surrounding pixels from only the dominant neighboring region in the interpolation of contour pixels is made to prevent smearing and blurring of contour areas in the final reconstructed image. The transition between neighboring segments is made bit more abrupt [9], but we avoid the distorting smearing of colors in contour areas.

The block diagrams for the encoder and decoder are given in Fig. 1. 


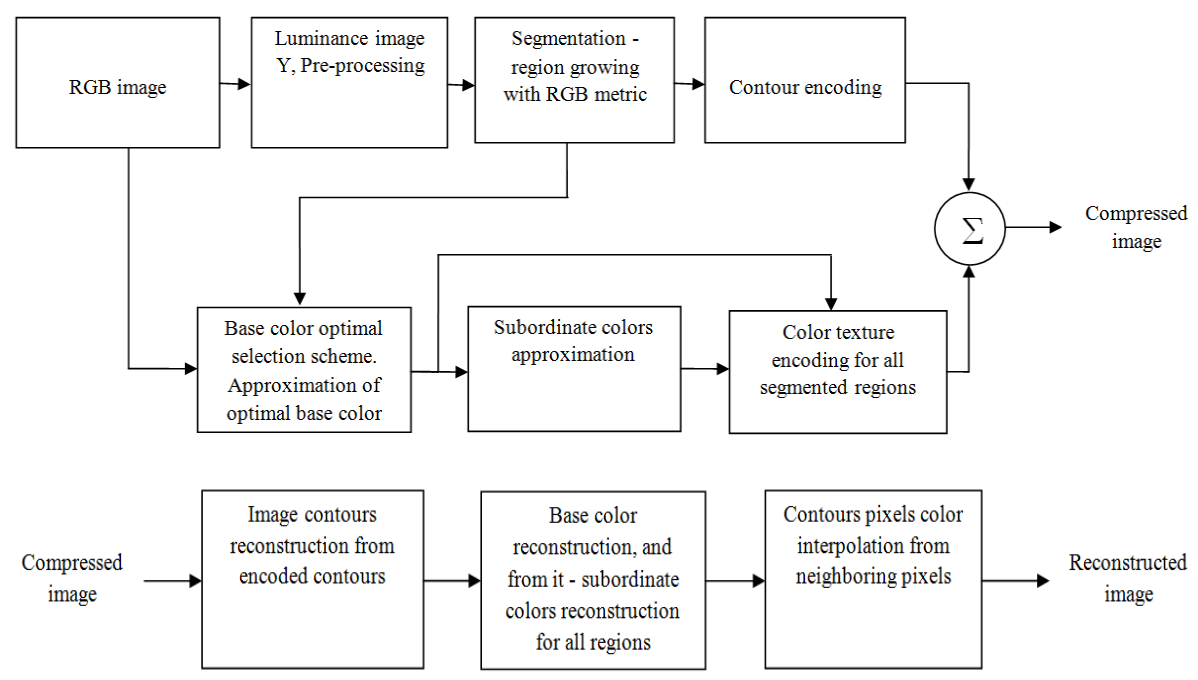

Fig. 1. - Block diagrams of the encoder (top) and decoder (bottom)

\section{Application Details and Results}

Our main proposed developments to improve the quality of reconstructed images are:

(1) Region Growing segmentation using full color information (Euclidean metric in RGB color space), rather than absolute distance in gray levels.

(2) An optimization scheme for the selection of an optimal base color for each segmented region.

To demonstrate the superiority of the reconstructions attained from the compression of color images using the above developments, we examine the compression results on the images "Peppers", "SolidWorks" and "Lena".

The parameters and thresholds are chosen as following: for Region Growing, the gray-level interval threshold [10] for merging a pixel to a region is chosen as 10 (/ 256). In RGB Region Growing, the Euclidean distance threshold is reasonably chosen approximate to $\sqrt{3 \cdot 10^{2}}=17.32$. The region size threshold for merging small regions is chosen as 10 pixels. The above parameters can be changed, to compare compressions with same compression ratios by PSNR measure.

In Fig. 2, the reconstructed images of the different compression procedures options are displayed, for the "Peppers" image. Here 'GL' stands for gray level; 'Optimal' refers to optimal base color selection. The areas of the image containing perceptually discernible distortions between the reconstructed images and the original image are mainly the transition areas between the red and green peppers and the green pepper's stalk. This distortion is significantly reduced by the suggested approach, as can be 
observed from Fig. 2(e), with RGB region growing and optimal base color. It is also noted that in addition to improvement in PSNR, optimal base color selection can lead to an increase in compression ratio (Fig. 2(d) and (e)), due to the possibility of approximation of regions' colors textures by lower degree polynomials, thus leading to fewer coefficients required for the encoding. An improvement in PSNR by more than 1 [dB] is acquired by RGB Region Growing and optimal base color selection. Fig. 2(f) displays JPEG2000 compression, for same ratio as in 2(e). For this image, which exhibits a degree of smoothness, the JPEG2000 is superior to our method by PSNR measure. Visually, however, JPEG2000 produces a result that is somewhat blurred.

The "SolidWorks" image was also examined, and a comparison of reconstruction results to JPEG [11] and JPEG2000 [12] compression algorithms was made.

The difference between Figs. 3(b), 3(c) is very distinct. In Fig. 3(b) the colors of some parts of the solid are distorted, and these artifacts are diminished in Fig. 3(c). Fig. 3(d) displays the JPEG compression result, which produces lower PSNR than our method (5 $[\mathrm{dB}]$ difference) and much lower compression ratio than our algorithm. Fig. 3(f) displays the results of JPEG2000 compression algorithm for the same compression ratio as in Fig. 3(c). Our method produces higher PSNR by more than 1 [dB]. This improvement exists in such structured images, but may be less pronounced for finely detailed, highly-textured images.

a

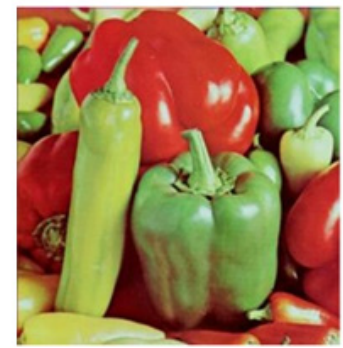

d

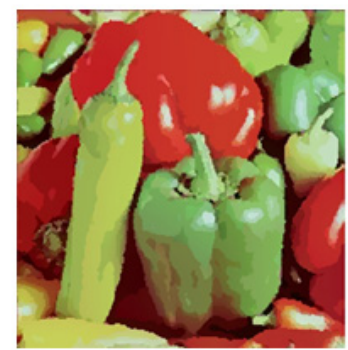

b

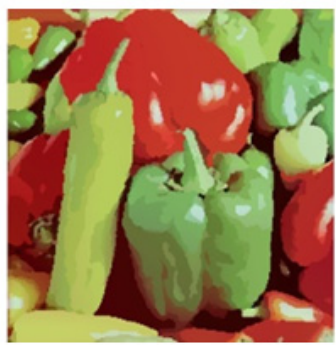

e

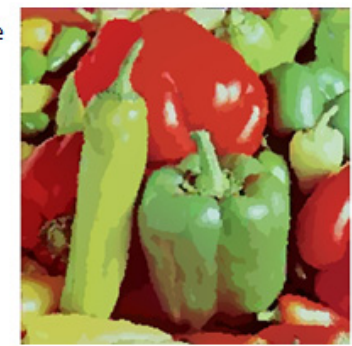

C

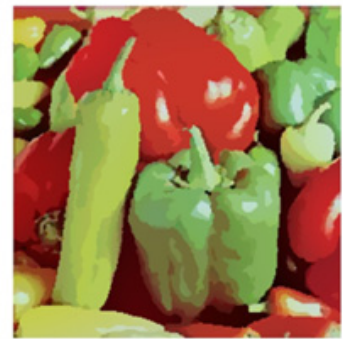

f

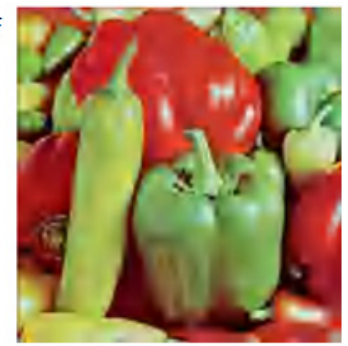

Fig. 2. Example of color image compression reconstruction results. (a) Original image.

(b) Region Growing (GL), base color (G) PSNR = 24.4332 [dB], compression ratio 89.

(c) Region Growing (GL), base color (Optimal) PSNR = 24.7226 [dB], compression ratio 92.

(d) Region Growing (RGB), base color (G) PSNR $=25.3185[\mathrm{~dB}]$, compression ratio 92.

(e) Region Growing (RGB), base color (Optimal) PSNR = 25.4374[dB], compression ratio 94 .

(f) JPEG2000 compression result, PSNR = 27.6061, compression ratio 94. 
Fig. 4 presents the algorithm sample run on the image "Lena", with highly detailed texture [13] in the hair area. The visual improvement between images 4(b) and 4(c) is perceptually noticeable - the smearing effects around the eyes and mouth in Fig. 4(b) are reduced in Fig. 4(c).

Table 1 contains information regarding the segmentation and compression of the presented images, when using RGB region growing and optimal base color selection procedure, e.g. number of segmented regions, contours properties etc.

Table 1. Segmentation and compression information for presented images

\begin{tabular}{|c|c|c|c|c|c|}
\hline image & $\begin{array}{c}\text { Number of } \\
\text { regions }\end{array}$ & $\begin{array}{c}\text { Avg. num. } \\
\text { Coefficients - } \\
\text { Green }\end{array}$ & $\begin{array}{c}\text { Avg. num. } \\
\text { Coefficients - } \\
\text { Red }\end{array}$ & $\begin{array}{c}\text { Avg. num. } \\
\text { Coefficients - } \\
\text { Blue }\end{array}$ & $\begin{array}{c}\text { Avg. contour } \\
\text { length [pixels] }\end{array}$ \\
\hline Peppers & 1329 & 2.51 & 1.97 & 2.32 & 18.18 \\
\hline SolidWorks & 356 & 2.14 & 1.86 & 2.4 & 10.91 \\
\hline Lena & 1253 & 2.63 & 1.85 & 2.22 & 21.14 \\
\hline
\end{tabular}

a
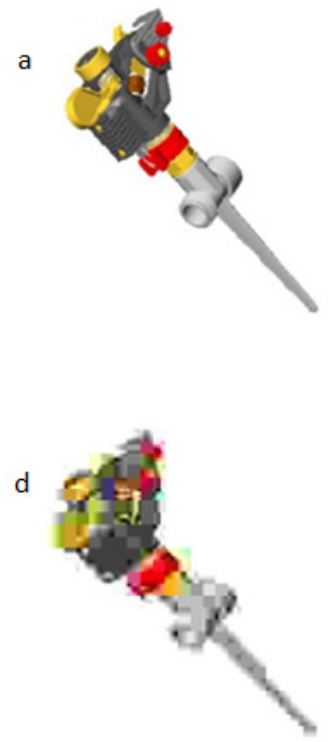

b
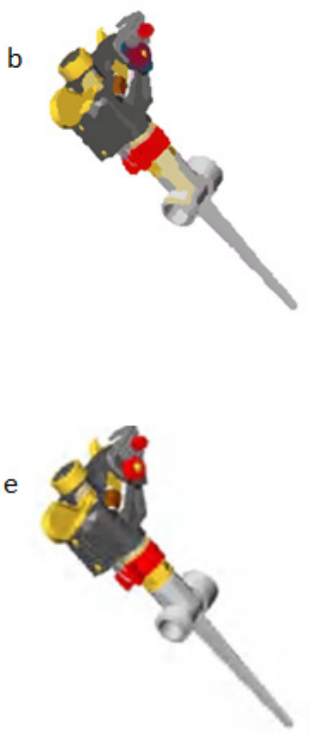

c
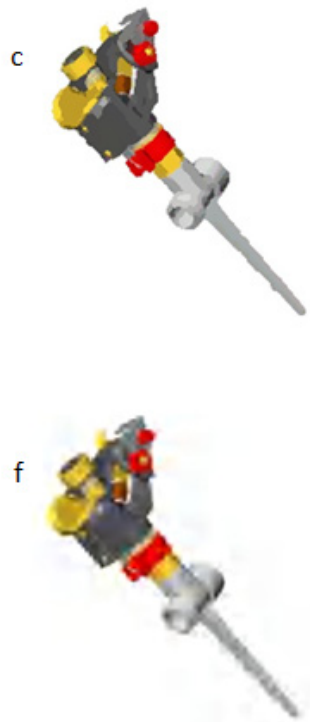

Fig. 3. A second example of color image compression reconstruction. (a) Original image.

(b) Region Growing (GL), base color (G) PSNR = 27.0026 [dB], compression ratio 190.

(c) Region Growing (RGB), base color (Optimal) PSNR = 29.2514[dB], compression ratio 190.

(d) JPEG compression, PSNR = $24.2[\mathrm{~dB}]$, compression ratio 85.

(e) JPEG2000 compression, PSNR $=29.88$ [dB], compression ratio 85 .

(f) JPEG2000 compression, PSNR = 28.16 [dB], compression ratio 190 (same as 3c). 
a

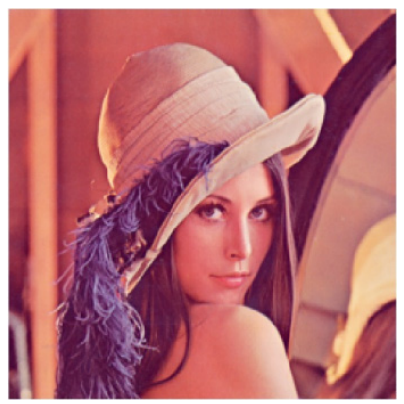

b

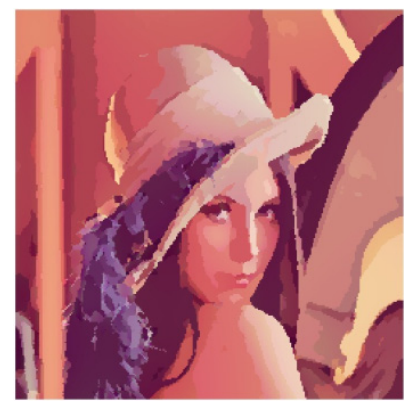

c

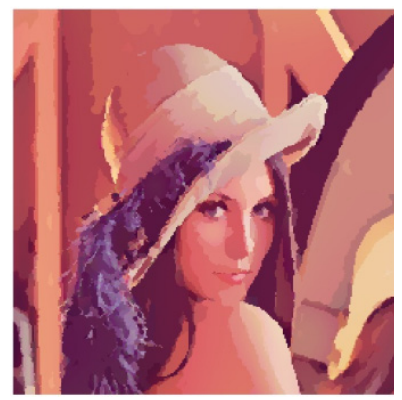

Fig. 4. Another example of color image compression reconstruction. (a) Original image.

(b) Region Growing (GL), base color (G) PSNR = 26.0021 [dB], compression ratio 81 .

(c) Region Growing (RGB), base color (Optimal) PSNR = 26.7238 [dB], compression ratio 83.

RGB Region Growing produces segmentation maps that are more detailed and more correlating to the human observer's perception of segmentation. RGB segmentation, compared to gray-level segmentation, is more computationally consuming. The compression ratios of the suggested algorithm vary in the range 30-90 for typical images, and can go up to 200 for structured images with well defined color regions.

\section{Summary and Conclusions}

In this work, we have expanded and developed existing methodologies of color image compression using inter-color correlation between the color primaries. Past works have dealt with exploiting this inter-correlation by expressing some color components as a functional of a base component, thus reducing the amount of data required for representation. There has also been use of image regions acquired from segmentation, instead of $\mathrm{NxN}$ square blocks. We suggested making full use of the color information in the Region Growing segmentation process, and implementing an optimization [14] procedure for the selection of a unique base color for each image region, which minimizes the colors' reconstruction errors (MSE) for that region. The above methodology can produce reconstructed results with higher quality than JPEG, for high compression ratios. It can also produce superior results compared to JPEG2000 compression standard for images of well-defined separate color regions.

These two main developments have been shown to improve compression reconstruction quality, each by its own, and together, both perceptually and by PSNR measure, with an increase in PSNR of more than $1[\mathrm{~dB}]$ for examined images.

Performing RGB Region Growing, instead of gray level, increases the compression PSNR measure significantly. Optimal base color selection can generally lead to an increase in compression ratio, by approximating regions' color textures of base and subordinate colors by lower degree polynomial functions, thus lowering the amount of bits required for encoding the color texture of an image. Our conclusion is that the proposed approach could improve the performance of presently available color compression methods. 
Acknowledgment. This research was supported in part by the Ollendorff Minerva Center. Minerva is funded through the BMBF.

\section{References}

1. Goffman-Vinopal, L., Porat, M.: Color image compression using inter-color correlation. In: Proceedings of the International Conference on Image Processing 2002, vol. 2, pp. II353-II-356 (2002)

2. Kotera, H., Kanamori, K.: A novel coding algorithm for representing full color images by a single color image. J. Imag. Technol. 16, 142-152 (1990)

3. Brice, C.R., Fennema, L.: Scene analysis using regions. Artif. Intell. (1), 205-226 (1970)

4. Kunt, M., Ikonomopoulos, A., Kocher, M.: Second-generation image-coding techniques. Proc. IEEE 73(4), 549-573 (1985)

5. Vedaldi, A., Soatto, S.: Quick Shift and Kernel Methods for Mode Seeking. In: Forsyth, D., Torr, P., Zisserman, A. (eds.) ECCV 2008, Part IV. LNCS, vol. 5305, pp. 705-718. Springer, Heidelberg (2008)

6. Wang, D.C.C., Vaganucci, A.H.: Gradient inverse weighted smoothing scheme and evaluation of performance. Comput. Graph. Image Process. 15 (1981)

7. Roterman, Y., Porat, M.: Color image coding using regional correlation of primary colors. Image and Vision Computing 25, 637-651 (2007)

8. Freeman, H.: On the encoding of arbitrary geometric configuration. IRE Trans. Electron. Comput. EC-10, 260-268 (1961)

9. Porat, M., Zeevi, Y.Y.: The generalized Gabor scheme of image representation in biological and machine vision. IEEE Transactions on Pattern Analysis and Machine Intelligence (PAMI) 10(44), 452-468 (1988)

10. Eldar, Y., Lindenbaum M., Porat M., Zeevi Y.Y.: The farthest point strategy for progressive image sampling. IEEE Transactions on Image Processing 6(9), 1305-1315 (1997)

11. Wallace, G.K.: The JPEG still picture compression standard. IEEE Transactions on Consumer Electronics 38(1), xviii-xxxiv (1992)

12. Christopoulos, C., Skodras, A., Ebrahimi, T.: The JPEG 2000 Still Image Coding System: An Overview. IEEE Transactions on Consumer Electronics 46(4), 1103-1127 (2000)

13. Nemirovsky, S., Porat, M.: On texture and image interpolation using Markov models. Signal Processing: Image Communication 24(3), 139-157 (2009)

14. Genossar, T., Porat, M.: Optimal bi-orthonormal approximation of signals. IEEE Transactions on Systems, Man and Cybernetics 22(3), 449-460 (1992) 